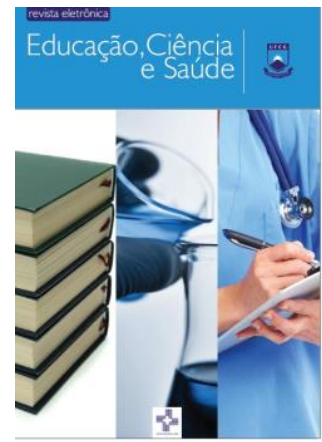

EDUCAÇÃO CIÊNCIA E SAÚDE

http://dx.doi.org/10.20438/ecs.v5i1.141

\title{
AVALIAÇÃO TOXICOLÓGICA FRENTE AS LARVAS DA ARTEMIA SALINA LEACH DO 1,2:3,4-DI-O-ISOPROPILIDENO- ALFA-D-GALACTOPIRANOSE
}

Antônio Ruan Souto dos Santos ${ }^{1}$, Herbert Igor Rodrigues de Medeiros ${ }^{2}$, Jonh Anderson Macêdo Santos ${ }^{3}$, Fernando de Sousa Oliveira ${ }^{4}$, Juliano Carlo Rufino Freitas $^{5}$

${ }^{1}$ Curso de Licenciatura em Química, Unidade Acadêmica de Biologia e Química, Universidade Federal de Campina Grande, Cuité-PB, Brasil.

${ }^{2}$ Curso de Bacharelado em Farmácia, Unidade Acadêmica de Saúde, Universidade Federal de Campina Grande, Cuité-PB, Brasil.

${ }^{3}$ Curso de Pós-graduação em Química da Universidade Federal Rural de Pernambuco, Departamento de Química, Universidade Federal Rural de Pernambuco, Recife-PE, Brasil.

${ }^{4}$ Professor da Unidade Acadêmica de Saúde, Universidade Federal de Campina Grande, Cuité-PB, Brasil.

${ }^{5}$ Professor da Unidade Acadêmica de Biologia e Química, Universidade Federal de Campina Grande, Cuité-PB, Brasil.

Email para correspondência: julianocrf@gmail.com

\section{Resumo}

O presente estudo teve como foco avaliar a toxicidade sobre a Artemia salina Leach do composto 1,2:3,4-di-O-isopropilideno-alfa-D-galactopiranose. Este composto foi sintetizado a partir da reação de acetalização da D-galactose, sendo posteriormente solubilizado em dimetilsulfóxido e água salgada artificial para obter as concentrações que foram adicionadas em tubos contentam naúplios da Artemia salina (10 unidades cada). Uma solução de dimetilsulfóxido $1 \%$ e dicromato de potássio foram utilizadas como controle negativo e positivo, respectivamente. As amostras foram submetidas à iluminação artificial durante $24 \mathrm{~h}$, e após este período de contato foram contabilizadas as larvas vivas e mortas. O cálculo da $\mathrm{CL}_{50}$ foi feito utilizando-se o programa POLO-PC. O 1,2:3,4-di-O-isopropilidenoalfa-D-galactopiranose foi obtido com $81 \%$ de rendimento e apresentou uma CL50 de $2.300,020 \mu \mathrm{g} / \mathrm{mL}$, com $95 \%$ de confiança. Este resultado da $\mathrm{CL}_{50}$ indica que o composto apresenta baixa toxicidade, e fornece subsídios para futuras pesquisas que visem sua aplicação biológica ou farmacológica.

Palavras-chave: Síntese Orgânica, Avaliação Toxicológica, Carboidrato, Artemia salina. 


\begin{abstract}
The present study aimed to evaluate on Artemia salina Leach the toxicity of the compound 1,2:3,4-di-O-isopropylidene-alpha-D-galactopyranose. This compound was synthesized from the acetalation reaction of D-galactose and then solubilized in dimethyl sulfoxide and artificial salt water to obtain the concentrations that were added to tubes containing Artemia salina nauplii (10 units each). A solution of $1 \%$ dimethyl sulfoxide and potassium dichromate were used as negative and positive control, respectively. The samples were submitted to artificial lighting for $24 \mathrm{~h}$, and after this contact period the live and dead larvae were counted. $\mathrm{LC}_{50}$ was calculated by using POLO-PC software. 1,2:3,4-Di-O-isopropylidene-alpha-Dgalactopyranose was obtained in $81 \%$ yield and showed an $\mathrm{LC}_{50}$ of $2,300.020$ $\mu \mathrm{g} / \mathrm{mL}$, with $95 \%$ confidence. This $\mathrm{LC}_{50}$ result indicates that the compound has low toxicity, and provides subsidies for future research aimed at its biological or pharmacological application.
\end{abstract}

Keywords: Organic Synthesis, Toxicological Evaluation, Carbohydrate, Artemia salina.

\title{
1 Introdução
}

As artêmias (Figura 1) são microcrustáceos encontrados em águas salinas, sendo capazes de viverem em ambientes extremamente salinos e com pouca concentração de oxigênio (RUIZ et al., 2005). De acordo com Camara e Castro (1983), as primeiras tentativas para disseminar a Artemia salina em ambientes naturais, foram realizadas no início da década de 60 , nas lagoas hipersalinas da Ilha Christmas, que em função das condições ecológicas adversas não foram bem-sucedidas. A ideia nesta disseminação era proporcionar condições favoráveis para a reprodução da Artemia salina, visando uma posterior liofilização das mesmas com o intuito de servir de alimentação para peixes de pequeno porte (peixes ornamentais). Este microcrustáceo é um ser filtrador que se alimenta de algas unicelulares, pequenos protozoários, bactérias e detritos dissolvidos no meio. A filtração ocorre nos toracópodos, que são encarregados de transportar as partículas alimentícias na direção do sistema digestivo, onde há uma redução da taxa de filtração, sem aumentar a concentração das partículas, pois ficarão acumuladas e irão interferir no processo fisiológico de seus batimentos (SOUTO, 1991). 


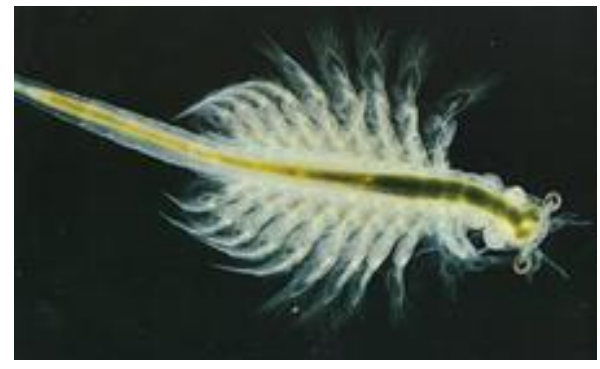

Figura 1. Imagem da Artemia Salina.

Fonte: imagem da artêmia - https://br.pinterest.com/explore/artemia-salina/

$\mathrm{Na}$ região Nordeste do Brasil, essas espécies de microcrustáceos chegaram através de inoculações feitas com seus cistos importados da Califórnia-EUA, em 1977, em Macau-RN, a partir daí as Artemia salinas se dispersaram por toda região salineira do Rio Grande do Norte e posteriormente da Região Nordeste (CAMARA; CASTRO, 1983).

A literatura descreve a utilização das artêmias para avaliação do efeito citotóxico de extratos (MOREIRA et al., 2002); ensaios citotóxicos de vegetais, mais especificamente de plantas medicinais (MEYER et al., 1982); teste de letalidade em algas (LHULLIER; HORTA; FALKENBERG, 2006); detecção de compostos bioativos em extratos vegetais como também expressar a toxicidade (RUIZ et al., 2005); e estudo fitoquímico (NASCIMENTO et al., 2008).

De acordo com Pimentel e colaboradores (2011), a literatura tem relatado correlações entre o bioensaio de toxicidade utilizando Artemia salina e a citotoxicidade em células humanas de tumores sólidos, e atividade frente ao antitripanossoma cruzi. Diante do exposto, as artêmias vêm sendo utilizadas na química medicinal por exemplo, para avaliar a toxicidade de compostos utilizados no combate as cepas de Aspergillus versicolor (MIAO, et al. 2012). $\mathrm{Na}$ área de síntese, vários estudos utilizaram as artêmias para a determinação da toxicidade in vitro dos compostos sintetizados (CHOHAN, et al. 2010). $\mathrm{Na}$ parte farmacológica e farmacêutica, observou-se que o uso deste crustáceo serviu de screening de produtos naturais visando a sua aplicação como: moluscicida, larvicida (LUNA, et al. 2005), anticonvulsivante (BAGHERI, et al. 2010), dentre outros. Seja em termos de precisão, ética, sensibilidade e simplicidade, este bioensaio tem superado amplamente o método com 
camundongos, e mais, recentemente foi comprovada também sua efetividade na detecção de toxinas produzidas por cianobactérias (RODRIGUEZ, et al., 2004). As artêmias são frequentemente utilizadas para testes de toxicidade (LIBRALATO et al., 2016), incluindo em testes utilizando carboidratos, como neste trabalho.

Os carboidratos são os compostos mais abundantemente encontrados em fontes naturais, e, assim como as proteínas, os lipídios e os ácidos nucleicos, são macromoléculas com estrutura polifuncional (FERREIRA, et al., 2009; BANDERA et al., 2014). Devido a esta abundância e suas diversas aplicações em várias áreas, é justificável o crescente aumento de artigos científicos, livros e grupos de pesquisas estudando a química e a síntese de carboidratos.

Nos últimos 100 anos, o estudo de suas atividades tem aumentado de forma surpreendente, principalmente com relação à suas aplicações biológicas (NOGUEIRA; PARMANHAN; CORREA, 2009). Dentre às suas atividades, estão: antineoplásico, antibacteriano, inseticida, antiviral, antifúngico, antiinflamatório, dentre outros (PUTERCA, et al., 2003). Com essas funções, os carboidratos têm atraído bastante 0 interesse de pesquisadores, particularmente dos químicos sintéticos, que buscam na modificação estrutural, um recurso para potencializar as atividades biológicas e farmacológicas já existentes. Consequentemente, os carboidratos têm sido submetidos a diferentes tipos de transformações, como a reação de acetilação (NIGUDKAR; DEMCHENKO, 2015), uma vez que, este tipo de reação é largamente utilizada pelos químicos sintéticos (YU, et al., 1999), onde é através dessas reações que obtêm-se compostos mais complexos a partir de compostos mais simples, como neste trabalho.

No geral, os testes utilizando a Artemia salina são eficazes, e vários grupos de pesquisa utilizam a metodologia proposta por Meyer e colaboradores (1982). Deste modo, o trabalho descreve a avaliação da toxicidade do composto 1,2:3,4-di-O-isopropilideno-alfa-D-galactopiranose utilizando a Artemia salina Leach. 


\section{Metodologia}

\subsection{Local de Pesquisa}

A etapa de síntese, purificação e o bioensaio da Artemia Salina Leach do 1,2:3,4-di-O-isopropilideno-alfa-D-galactopiranose (2) foram realizadas no Laboratório de Síntese Orgânica (LASO) e no Laboratório de Toxicologia, ambos localizados no Centro de Educação e Saúde da Universidade Federal de Campina Grande - Campus Cuité/PB. Enquanto que, a etapa de caracterização do 1,2:3,4-di-O-isopropilideno-alfa-D-galactopiranose (2), ou seja, as análises de rotação específica e os espectros de infravermelho (IV) e ressonância magnética nuclear (RMN) de hidrogênio $\left({ }^{1} \mathrm{H}\right)$ e carbono $\left({ }^{13} \mathrm{C}\right)$ foram realizados na Central Analítica da Universidade Federal do Pernambuco Campus Recife/PE.

\subsection{Materiais e Equipamentos}

Em geral, foram utilizados reagentes e solventes na sua forma comercial, dos fornecedores Sigma-Aldrich, Dinâmica e Cinética. Os solventes utilizados foram previamente purificados e secos conforme o protocolo descrito por Perrin e Armarego (1996), no qual, o hexano e o acetato de etila (AcOEt) foram destilados utilizando coluna de Vigreux, enquanto que a propanona foi destilada sob iodeto de sódio.

Para o acompanhamento da evolução e término da reação, foi utilizada a cromatografia em camada delgada (CCD) utilizando como sistema eluente hexano:acetato de etila na proporção de 60:40, respectivamente. Após a eluição cromatográfica da CCD, a mesma foi revelada utilizando uma solução etanólica ácida [solução contendo etanol e ácido sulfúrico na proporção de 95:5, respectivamente] sob aquecimento.

O solvente da solução contendo o 1,2:3,4-di-O-isopropilideno-alfa-Dgalactopiranose (2) foi evaporado utilizando um evaporador rotativo da Visatom conectado a uma bomba de vácuo New PumsSparmaz, enquanto que o solvente residual foi removido através de uma bomba de alto vácuo da ColeParmer.

Os espectros de RMN de ${ }^{1} \mathrm{H}$ e ${ }^{13} \mathrm{C}$ do 1,2:3,4-di-O-isopropilideno-alfa-Dgalactopiranose (2) foram registrados em um espectrômetro Varian URMNS de 
$400 \mathrm{MHz}$, no qual, os deslocamentos químicos estavam expressos em partes por milhão (ppm) em relação ao pico residual do clorofórmio (7,26 ppm) para os espectros de hidrogênio, em relação ao pico central do clorofórmio deuterado (77,0 ppm) para os espectros de carbono. No mais, todas as constantes de acoplamento $(\mathcal{C})$ estão descritas em hertz $(\mathrm{Hz})$.

O espectro de IV do 1,2:3,4-di-O-isopropilideno-alfa-D-galactopiranose (2) foi realizado em um espectrofotômetro de infravermelho com transformada de Fourier no Spectrum 400 FT-IR/FT-NIR Spectrometer modelo PerkinElmer, enquanto que, a sua rotação específica foi determinada em um polarímetro digital da marca JASCO ${ }^{\circledR}$ P-2000 equipado com a luz de sódio em comprimento de onda $589 \mathrm{~nm}$. A amostra foi preparada em concentração de $1 \mathrm{~m} / \mathrm{v} \%$ (em $\mathrm{MeOH}$ ) em uma cubeta de $1 \mathrm{~mL}$.

\subsection{Procedimento Experimental}

\subsubsection{Síntese do 1,2:3,4-di-O-isopropilideno-alfa-D-galactopiranose (2)}

Em um balão de fundo redondo de capacidade de $50 \mathrm{~mL}$, contendo $15 \mathrm{~mL}$ de propanona seca e destilada, foram adicionados a D-galactose anidra (1, 0,9 g, $5 \mathrm{mmol})$, sulfato de cobre anidro (2 g, 12,5 mmol) e 0,1 $\mathrm{mL}$ de ácido sulfúrico concentrado (Cuidado: o ácido deve ser adicionado lentamente, por se tratar de um agente desidratante). A mistura reacional foi agitada por 36 horas em temperatura ambiente $\left(28 \pm 2^{\circ} \mathrm{C}\right)$ e sob atmosfera inerte. Uma vez comprovado 0 término da reação por CCD, o sulfato de cobre foi removido por filtração a vácuo e lavado com propanona $(2 \times 15 \mathrm{~mL})$. Na sequência, neutralizou-se a fase orgânica com solução aquosa de hidróxido de cálcio $0,1 \mathrm{M}(5 \mathrm{~mL})$. A esta mistura foi adicionado $20 \mathrm{~mL}$ de acetato de etila $(20 \mathrm{~mL})$ e separou-se a fase orgânica. A fase orgânica foi seca sob sulfato de magnésio anidro, o solvente evaporado sob pressão reduzida e o 1,2:3,4-di-O-isopropilideno-alfa-Dgalactopiranose (2) purificado por cromatografia em coluna utilizando como solvente eluente apenas o hexano terminado com o sistema eluente hexano:acetato de etila na proporção de 75:25, respectivamente. 


\subsubsection{Bioensaio Toxicológico com as Larvas da Artemia Salina Leach}

O bioensaio toxicológico com Artemia Salina Leach foi baseado no protocolo descrito por Meyer e colaboradores (1982). Foram utilizados $30 \mathrm{mg}$ do 1,2:3,4di-O-isopropilideno-alfa-D-galactopiranose (2), o qual foi adicionado $50 \mu \mathrm{L}$ de dimetilsulfóxido (DMSO). A solução foi homogeneizada e o volume completado para $5 \mathrm{~mL}$ com água salinizada filtrada (água salina e água destilada 1:1) a pH = 8,0. Desta solução foram retiradas alíquotas de 2500, 2291, 2083, 1875, 1666, 1458 e $1250 \mu \mathrm{L}$ que foram transferidas para outros balões volumétricos de $5 \mathrm{~mL}$ e os volumes completados com o mesmo solvente, obtendo-se concentrações de 3000, 2750, 2500, 2250, 2000, 1750 e $1500 \mu \mathrm{g} / \mathrm{mL}$, para a solução aquosa de 1,2:3,4-Di-O-isopropilideno-alfa-D-galactopiranose. O teste foi acompanhado por controle positivo onde foi utilizado o dicromato de potássio $\left(\mathrm{K}_{2} \mathrm{Cr}_{2} \mathrm{O}_{7}\right)$ e controle negativo utilizando apenas água marinha artificial e DMSO (concentração e 1\% v/v). Os cistos de Artemia salina (20 mg) foram incubados sob iluminação artificial por $24 \mathrm{~h}$ para que favorecesse a eclosão das larvas (metanáuplios), e estas, separadas em nove grupos com 10 artêmias cada. O primeiro e o segundo grupo recebeu a solução controle positivo (solvente e dicromato de potássio) e controle negativo (solvente e DMSO, respectivamente) e as sete seguintes receberam a solução aquosa do composto em diferentes concentrações. As amostras foram submetidas à iluminação artificial durante $24 \mathrm{~h}$, e após este período foram contabilizadas as larvas mortas. $O$ experimento foi realizado em triplicata para cada substânciateste.

No que se refere ao teste de letalidade com as artêmias, as mesmas foram colocadas em ambientes similares ao ambiente natural, com a salinidade elevada e baixas concentrações de oxigênio, onde a substância colocada foi diluída, a partir daí, após um dia, se teve a contagem de larvas mortas como também vivas, para assim, se ter o teste de letalidade realizado em sua totalidade, através de analises estatística e como também análises comparativas com resultados já consolidados (LHULLIER; HORTA; FALKENBERG, 2006; MOREIRA et al., 2002). 


\subsubsection{Análise estatística}

Para obtenção dos valores da concentração letal que mata $50 \%$ das larvas $\left(\mathrm{CL}_{50}\right)$, foi utilizada a análise através do software POLO-PC (Copyright LeOra Software 1987) com $95 \%$ de confiança.

\section{$3 \quad$ Resultados}

Visando avaliar a toxicidade do 1,2:3,4-di-O-isopropilideno-alfa-Dgalactopiranose (2), o trabalho foi iniciado com a síntese deste composto através da reação de acetalização da D-galactose (1) pela propanona em meio ácido, conforme descrito na figura 2 (DANTAS et al., 2017).
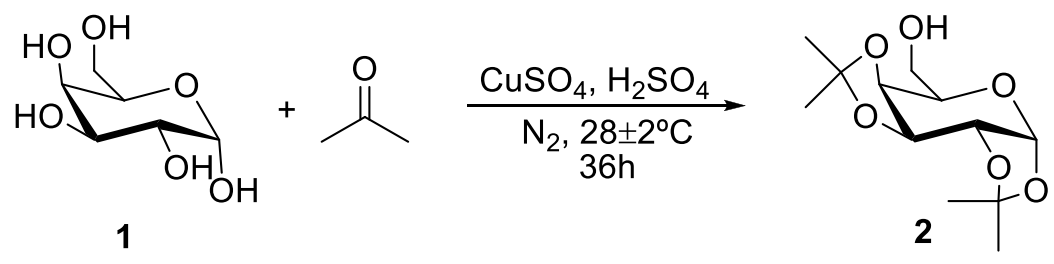

Figura 2. Reação de acetalização da D-galactose (1).

O 1,2:3,4-di-O-isopropilideno-alfa-D-galactopiranose (2) apresentou aspecto oleoso, viscoso e de colocação amarelada. Após a etapa de purificação, este composto, foi obtido com $81 \%$ de rendimento, sendo este resultado um pouco superior ao descrito na literatura (SCHMIDT, 1963). Adicionalmente, este composto foi caracterizado por diferentes técnicas espectroscópicas, tais como: rotação específica, infravermelho e ressonância nuclear, onde estes dados espectroscópicos estão sumarizados na Tabela 1. 
Tabela 1: Informações espectroscópicos do 1,2:3,4-di-O-isopropilideno-a-Dgalactopiranose (2)

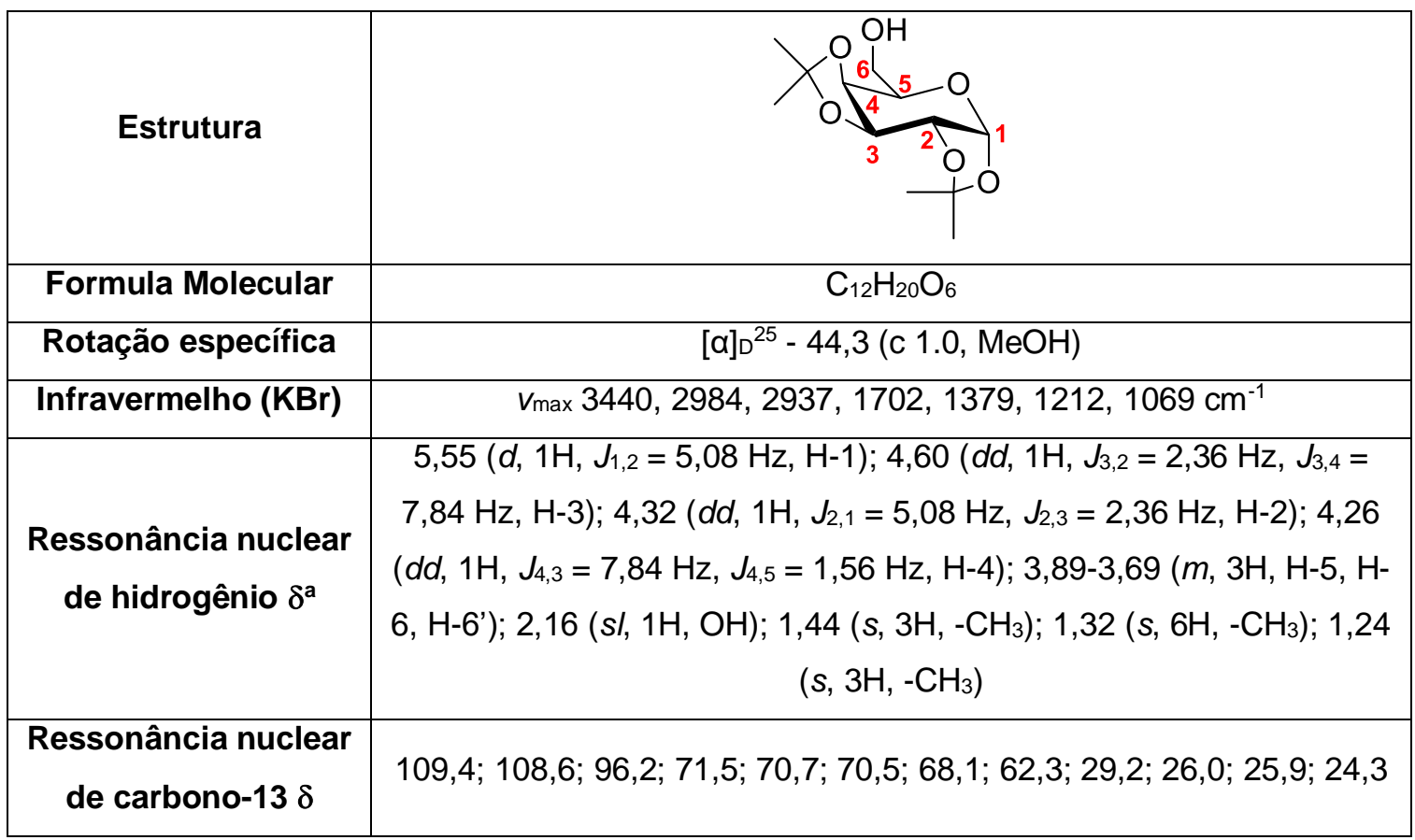

aSímbolos: $\delta$ = deslocamento químico; $\mathrm{J}=$ constate de acoplamento; $\mathrm{s}=$ simpleto; $\mathrm{sl}=$ simpleto largo; $\mathrm{d}$ = dupleto; $\mathrm{t}=$ tripleto; qua = quarteto;

Uma vez sintetizado e devidamente caracterizado o 1,2:3,4-di-Oisopropilideno-alfa-D-galactopiranose (2), iniciou-se o bioensaio toxicológico frente às larvas de Artemia salina Leach. Deste modo, os valores da taxa de mortalidade do composto 2 variaram entre 0 e 100\%, visando deste modo, determinar a concentração letal que mata $50 \%$ das larvas $\left(C_{50}\right)$.

Cabe salientar que este bioensaio foi realizado de acordo com o protocolo de Mayer (1982) com algumas modificações, onde foram testadas as concentrações de 3000, 2750, 2500, 2250, 2000, 1750 e $1500 \mu \mathrm{g} / \mathrm{mL}$ do composto 2, e realizado o controle positivo e negativo. Para o controle negativo foi utilizado uma solução aquosa de DMSO, uma vez que, este solvente foi utilizado para solubilizar o composto 2 , enquanto que o controle positivo foi realizado com $\mathrm{K}_{2} \mathrm{Cr}_{2} \mathrm{O}_{7}$ como descrito por Belem e colaboradores (2015).

A CL50 encontrada através do software POLO-PC, com 95\% de confiança, foi de 2.300,020 $\pm 57,408 \mu \mathrm{g} / \mathrm{mL}$ com intervalo nos limites de 2.242,612 a 2.357,428 $\mu \mathrm{g} / \mathrm{mL}$. De acordo com Merino e colaboradores (2015), este valor da $\mathrm{CL}_{50}$ para o 1,2:3,4-di-O-isopropilideno-alfa-D-galactopiranose (2) implica que 0 composto é pouco tóxico, uma vez que esse valor se enquadra nos índices superiores a $1000 \mu \mathrm{g} / \mathrm{mL}$. No mais, o bioensaio do controle negativo levou a 
morte de nenhuma larva, enquanto que a $\mathrm{CL}_{50}$ do controle positivo foi semelhante ao descrito por Belém et al. (2015).

Este resultado da $\mathrm{CL}_{50}$ para o 1,2:3,4-di-O-isopropilideno-alfa-Dgalactopiranose (2) serve como motivação para futuros estudos envolvendo a aplicação desse composto no setor farmacológico, uma vez que, segundo Devulapalle e colaboradores (2004), derivados de carboidratos são promissores na prevenção contra cáries e de higiene oral para o combate da placa bacteriana, além de outras aplicações.

\section{Conclusão}

Em suma, neste estudo foi descrito a síntese e avaliação toxicológica do 1,2:3,4-di-O-isoproleno-alfa-D-galactopiranose frente às larvas da Artemia salina Leach. O composto derivado da D-galactose foi obtido em bom rendimento (81\%). Apos a análise dos dados obtidos nos bioensaios, foi possível constatar que o 1,2:3,4-di-O-isoproleno- $\alpha$-D-galactopiranose apresentou baixa toxicidade frente às larvas da Artemia salina Leach apresentando um valor de $\mathrm{CL}_{50}$ de $2300,020 \mu \mathrm{g} / \mathrm{mL}$. Tal resultado, demonstra que o composto sintetizado tem boa aceitação biológica e encoraja nosso grupo de pesquisa a realizar novas modificações estruturais visando a sua aplicação na área farmacológica.

\section{$5 \quad$ Referências}

BAGHERI, S.; SAHEBKAR, A.; GOHARI, A.; SAEIDNIA, S.; MALMIR, M.; IRANSHAHI, M. Evaluation of cytotoxicity and anticonvulsant activity of some Iranian medicinal Ferula species. Pharmaceutical Biology, v. 48, n. 3, p. 242246, 2010.

BANDERA, D.; SAPKOTA, J.; JOSSET, S.; WEDER, C.; TINGAUT, P.; GAO, X.; FOSTER, E. J.; ZIMMERMANN, T. Influence of mechanical treatments on the properties of cellulose nanofibers isolated from microcrystalline cellulose. Reactive and Functional Polymers. v. 85, p. 134-141, 2014.

BELEM, V. A.; LIMA, F. G. C.; BARROS, L. M.; DUARTE A. E. Estudo da toxicidade e atividade antifungica de Lantana camara L. (Verbenaceae) como 
ferramenta de preservação da espécie. Caderno de Cultura e Ciência, ano IX, v. 13, n. 2, p. 51-59, 2015.

CAMARA, M. R.; CASTRO, E. V. Artemia salina I. (anostraca); uma opção para a aqui cultura do Nordeste do Brasil. Revista Brasileira de Zoologia. v. 1, n. 3 , p. $145-147,1983$.

CHOHAN, Z.; SUMRRA, S.; YOUSSOUFI, M.; HADDA, T. Metal based biologically active compounds: Design, synthesis, and antibacterial/antifungal/cytotoxic properties of triazole-derived Schiff bases and their oxovanadium(IV) complexes. European Journal of Medicinal Chemistry, v. 45, n. 7 , p. $2739-2747,2010$.

DANTAS, A. R. S.; MIRANDA, M. D. S.; SANTOS, J. A. M.; SANTOS, C. S.; SILVA, C. L. L.; FREITAS, J. C. R. Estudo e otimização das condições reacionais visando a síntese do 1,2:3,4-di-o-isopropilideno- $\alpha$-d-galactopiranose. Revista de Educação, Ciência e Saúde. v. 4, n. 1, p. 19-32, 2017.

DEVULAPALLE, K. S.; SEGURA, A. G.; FERRER, M.; ALCALDE, G. M.; PLOU, F. J. Effect of carbohydrate fatty acid esters on Streptococcus sobrinus and glucosyltransferase activity. Carbohydrate Research. v. 339, n. 6, p. 1029-1034, 2004.

FERREIRA, V. FRANCISCO; ROCHA, D. R.; SILVA, F. C. Potencialidades e oportunidades na química da sacarose e outros açúcares. Química Nova. v. 32, n. 3, p. 623-638, 2009.

RODRIGUEZ, A. G.; CAETANO, G. G. I.; BORGES, M. B.; SANTOS, C. A.; CARDOSO, G. S. Toxicidade por Microcystis sp. em Goiás: Estudo dos efeitos tóxicos em camundongos e Artemias salinas. Estudos (Goiania). v. 31, n. 9, p. 1595-1606, 2004. 
LHULLIER, C.; HORTA, P. A.; FALKENBER, M. Avaliação de extratos de macroalgas bênticas do litoral catarinense utilizando o teste de letalidade para Artemia salina. Revista Brasileira de Farmacognosia. v. 16, n. 2, p. 158-163 2006.

LiBRALATO, G. A.; PRATO, E.; MIGLIORE, L.; CICERO, A. M.; MANFRA, L. A review of toxicity testing protocols and endpoints with Artemia spp. Ecological Indicators. v. 69, p. 35-49, 2016.

LUNA, J. S; SANTOS, A. F; LIMA, M. R. F; OMENA, M. C; MENDONÇA, F. A. C; BIEBER, L. W; SANT'ANA, A. E. G. A study of the larvicidal and molluscicidal activities of some medicinal plants from northeast Brazil. Journal of Ethnopharmacology. v. 97, p. 199-206, 2005.

MERINO, F. J. Z.; OLIVEIRA, V. B.; PAULA, C. S.; CANSIAN, F. C.; SOUZA, A. M.; ZUCHETTO, M.; HIROTA, B. C. K.; DUARTE, A. F. S.; KULIK, J. D.; MIGUEL, M. D.; MIGUEL, O. G. Análise fitoquímica, potencial antioxidante e toxicidade do extrato bruto etanólico e das frações da espécie Senecio westermanii Dusén frente à Artemia salina. Revista Brasileira de Planta Médica. v. 17, n. 4, p.1031-1040, 2015.

MEYER, B. N.; FERRIGNI, n. R.; PUTNAM, J.E.; JACOBSEN, L. B.; NICHOLS, D. E.; MCLAUGHLIN, J. L. Brine Shimp: A conveniente general bioassay for active plant constituints. Journal of Medicinal Plant Research. v. 45, p. 31-34, 1982.

MIAO, F. P.; LI, X. D.; LIU, X. H.; CICHEWICZ, R. H.; JI, N. Y. Secondary Metabolites from an Algicolous Aspergillus versicolor Strain. Marine Drugs, v. 10, n. 1, p. 131-139, 2012.

MOREIRA, F. P. M.; COUTINHO, V.; MONTANHER, A. B. P.; CARO, M. S. B.; BRIGHENTE, I. M. C.; PIZZOLATTI, M. G. FLAVONÓIDES E TRITERPENOS 
DE Baccharis pseudotenuifolia - bioatividade sobre Artemia salina. Química Nova. v. 26, n.3, p. 309-311, 2002.

NASCIMENTO, J. E.; ALBUQUERQUE, U. P. Estudo fitoquímico e bioensaio toxicológico frente a larvas de Artemia salina Leach. de três espécies medicinais do gênero Phyllanthus (Phyllanthaceae). Revista de Ciências Farmacêuticas Básica e Aplicada. v. 29, p. 145-150, 2008.

NIGUDKAR, S. S.; DEMCHENKO, A. V. Stereocontrolled 1,2-cis glycosylation as the driving force of progress in synthetic carbohydrate chemistry. Chemical Science. v. 6, p. 2687-2704, 2015.

NOGUEIRA C. M.; PARMANHAN, B. R.; FARIAS P. P.; CORRÊA, A. G. A importância crescente dos carboidratos em química medicinal. Revista Virtual Química. v.1, n. 2, p. 149-159, 2009.

PERRIN, D. D.; AMAREGO, W. L. F. Purification of Laboratory Chemicals. Pergamon Press: Oxford. 1996.

PIMENTEL, M.F; JÚNIOR, F. C. G. S.; SANTAELLA, S. T.; LOTUFO, L. V. C. O Uso de Artemia sp. como Organismo-Teste para Avaliação da Toxicidade das Águas Residuárias do Beneficiamento da Castanha de Caju Antes e Após Tratamento em Reator Biológico Experimental. Journal of the Brazilian Society of Ecotoxicology. v. 6, n. 1, p. 15-22, 2011.

PUTERKA, G. J.; FARONE, W.; PALMER, T.; BARRINGTON, A. StructureFunction Relationships Affecting the Insecticidal and Miticidal Activity of Sugar Esters. Journal of Economic Entomology. v. 96, p. 636-644, 2003.

RUIZ, A. L. T.; MAGALhÃES, E. G.; MAGALhÃES, A. F.; FARIA, A. D.; AMARAL, M. C. E.; SERRANO, D. R.; MAGALHÃES, E. M. Z.; MAGALHÃES, L. A. Avaliação da atividade tóxica em Artemia salina e Biomphalaria glabrata 
de extratos de quatro espécies do gênero Eleocharis (Cyperaceae). Revista Brasileira de Farmacognosia. v. 15, n. 2, p. 98-102, 2005.

SCHMIDT, O. T.; In: Whistler, Roy L.; Wolfrom, M. L. ed.s. Methods in carbohydrate chemistry. Volume 2, Reactions of carbohydrates. Academic Press: New York, p. 318-325, 1963.

SOUTO, F. J. B. Influencias de parâmetros ambientais sobre Artemia sp (Branchiopoda: Artemiidae) em uma salina artesanal do estado do Rio Grande do Norte. Curso de Mestrado em Ciências Biológicas - Zoologia, Universidade Federal da Paraíba, 19 p. 1991.

YU, B.; XIE, J.; DENG, S.; HUI, Y. J. First Synthesis of a Bidesmosidic Triterpene Saponin by a Highly Efficient Procedure. Journal of the American Chemical Society. v. 121, n. 51, p. 12196-12197, 1999.

\section{Agradecimentos}

Os autores agradecem às agências de formento CNPq (447361/2014-7) ao PRONEM/FACEPE (APQ-0476-1.06/14) pelo suporte financeiro e a CAPES pelas bolsas concedidas. A Central Analítica do Departamento de Química Fundamental da Universidade Federal de Pernambuco pelas análises do composto sintetizado. 